Article

\title{
Representation of Maximal Surfaces in a 3-Dimensional Lightlike Cone
}

\author{
Jinhua Qian ${ }^{1}{ }^{1}$, Xueshan $\mathrm{Fu}^{2}$ and Huili Liu ${ }^{1, *}$ \\ 1 Department of Mathematics, Northeastern University, Shenyang 110004, China; qianjinhua@mail.neu.edu.cn \\ 2 Department of Mathematics, Jeju National University, Jeju 63243, Korea; xsfu@jejunu.ac.kr \\ * Correspondence: liuhl@mail.neu.edu.cn
}

Received: 6 October 2020; Accepted: 26 October 2020; Published: 2 November 2020

check for updates

\begin{abstract}
In this paper, the representation formula of maximal surfaces in a 3-dimensional lightlike cone $\mathbb{Q}^{3}$ is obtained by making use of the differential equation theory and complex function theory. Some particular maximal surfaces under a special induced metric are presented explicitly via the representation formula.
\end{abstract}

Keywords: complex function; lightlike cone; maximal surface; representation formula

\section{Introduction}

There are very special submanifolds in some event horizons of the compact Cauchy horizons of Taub-NUT, which are called null submanifolds due to the degenerate induced metrics. Null submanifolds often seem to be some smooth parts of the achronal boundaries in general relativity. In fact, the degenerate submanifolds of Lorentzian manifolds may be of great use to explore the intrinsic structure of manifolds with degenerate metrics.

It is well known that the pseudo Riemannian space forms are non-degenerate and complete pseudo Riemannian hypersurface with zero, positive, or negative constant sectional curvature, which are consisted by the pseudo Euclidean space $\mathbb{E}_{q}^{n}$, the pseudo Riemannian sphere $\mathbb{S}_{q}^{n}(c, r)$ and the pseudo Riemannian hyperbolic space $\mathbb{H}_{q}^{n}(c, r)$. Many research works have been done in the pseudo Riemannian space forms that are similar to those of Riemannian spacetime [1,2]. However, to some extent, the degenerate hypersurface $\mathbb{Q}_{q}^{n}(c)$ in $\mathbb{E}_{q}^{n+1}$ should be regarded as another kind of pseudo Riemannian space form or be called degenerate pseudo Riemannian space form. Some geometers and experts have studied the geometry of submanifolds in degenerate pseudo Riemannian space form [3-6]. Taking the trapped surface as an example, which is a compact, spacelike 2-dimensional submanifold of spacetime on which the divergence of the outgoing null vector orthogonal to the surface converges. Meanwhile, a spacelike submanifold is said to be a marginally trapped submanifold in the pseudo Euclidean space $\mathbb{E}_{q}^{n}$ when its mean curvature vector is lightlike at every point [5]. The notions of trapped surfaces and marginally trapped surfaces play important roles in general relativity and cosmology, which are the foundations of the Penrose inequality and the cosmic censorship hypothesis, etc. $[7,8]$. In 2009 , B. Y. Chen tested the cone surface of $\mathbb{Q}^{3}$ is marginally trapped in $\mathbb{E}_{1}^{4}$ iff it is flat [7], one of the authors of this paper proved that the surface in $\mathbb{Q}^{3}$ is flat if the surface is maximal $[3,6]$.

Based on the authors' previous works about the submanifolds in lightlike cones, we consider the maximal surfaces in a 3-dimensional lightlike cone in this work. This paper is organized as follows. At first, some basic knowledge is recalled including the definition of maximal surface and the solving method of particular differential equation, etc. Then we give a representation formula for maximal surfaces and construct some classical examples of maximal surfaces in $\mathbb{Q}^{3}$, which will lay solid foundation for related future research works. 


\section{Preliminaries}

Let $\mathbb{E}_{q}^{m}$ be the $m$ dimensional pseudo Euclidean space with respect to the metric

$$
\langle x, y\rangle=\sum_{i=1}^{m-q} x_{i} y_{i}-\sum_{j=m-q+1}^{m} x_{j} y_{j}
$$

where $x=\left(x_{1}, x_{2}, \ldots, x_{m}\right), y=\left(y_{1}, y_{2}, \ldots, y_{m}\right) \in \mathbb{E}_{q}^{m}$.

Let $p$ be a fixed point in $\mathbb{E}_{q}^{m}$. Then $\mathbb{S}_{q}^{n}(p, r)=\left\{x \in \mathbb{E}_{q}^{n+1}:\langle x-p, x-p\rangle=r^{2}\right\}$ is called the pseudo Riemannian sphere; $\mathbb{H}_{q}^{n}(p, r)=\left\{x \in \mathbb{E}_{q+1}^{n+1}:\langle x-p, x-p\rangle=-r^{2}\right\}$ is called the pseudo Riemannian hyperbolic space and $\mathbb{Q}_{q}^{n}(p)=\left\{x \in \mathbb{E}_{q}^{n+1}:\langle x-p, x-p\rangle=0\right\}$ is said to be the pseudo Riemannian lightlike cone, respectively.

Where $r>0$ is a constant, the point $p$ is said to be the center of $\mathbb{S}_{q}^{n}(p, r), \mathbb{H}_{q}^{n}(p, r)$ and $\mathbb{Q}_{q}^{n}(p)$. When $p$ is the origin and $q=1$, we denote $\mathbb{Q}_{1}^{n}$ by $\mathbb{Q}^{n}$ and call it the lightlike cone [3].

Definition 1 ([6]). Let $x: \mathbb{M}^{n} \rightarrow \mathbb{Q}^{n+1}$ be a non-degenerate hypersurface. Then the mean curvature $H$ of $x$ is defined as

$$
n H=\langle\Delta x, y\rangle .
$$

Especially, if $H \equiv 0$, then $x$ is called the maximal hypersurface.

In order to serve the following discussions, we recall some basic knowledge and solving method of differential equations.

Lemma 1. Let $F=F(z),(z \in D \in \mathbb{C})$ be a complex differentiable function. When

$$
\operatorname{Im} \frac{\partial F}{\partial \bar{z}} \equiv 0,
$$

the integral $\int \operatorname{Re} F d z$ does not depend on the path of the integration, and

$$
\frac{\partial}{\partial z} \int \operatorname{Re} F d z=\frac{1}{2} F(z)
$$

Proof. Let the complex differentiable function $F(z)$ be decomposed as

$$
F(z)=\operatorname{Re} F(z)+i \operatorname{Im} F(z)=\xi(z)+i \eta(z),
$$

then

$$
F d z=(\xi d u-\eta d v)+i(\eta d u+\xi d v) .
$$

The integral $\int \operatorname{Re} F d z$ does not depend on the path of the integration if and only if

$$
d(\xi d u-\eta d v)=-2 \operatorname{Im} \frac{\partial F}{\partial \bar{z}} d u \wedge d v \equiv 0
$$

And in this case, we have

$$
2 \frac{\partial}{\partial z}\left(\int \operatorname{Re} F d z\right)=\left(\frac{\partial}{\partial u}-i \frac{\partial}{\partial v}\right) \int(\xi d u-\eta d v)=\xi+i \eta=F(z) .
$$

Lemma 2. Given a differential equation, namely

$$
F_{t t}(t)=a(t) F(t)+b(t) F_{t}(t),
$$


then it has the following solution as

$$
F(t)=c \exp \left(\int\left[\sqrt{-\varphi} \frac{\cos \theta}{\sin \theta}+\frac{1}{2} b(t)\right] d t\right) \quad(c \in \mathbb{R}),
$$

where $\varphi(t)=a(t)+\frac{1}{4} b^{2}(t)-\frac{1}{2} b^{\prime}(t)$ and $\theta$ satisfies

$$
\theta^{\prime}(t)=\sqrt{-\varphi}+\frac{\varphi^{\prime}}{2 \varphi} \sin \theta \cos \theta
$$

Proof. By a transformation as $F(t)=f(t) e^{\frac{1}{2} \int b(t) d t}$, then the Equation (1) can be changed to

$$
f_{t t}(t)=\varphi(t) f(t),
$$

where $\varphi(t)=a(t)+\frac{1}{4} b^{2}(t)-\frac{1}{2} b^{\prime}(t)$.

Putting

$$
\left\{\begin{array}{l}
\rho \sin \theta=f \sqrt{-\varphi}, \\
\rho \cos \theta=f^{\prime}(t),
\end{array}\right.
$$

then we have

$$
\left\{\begin{array}{l}
\rho^{\prime} \sin \theta+\rho \theta^{\prime} \cos \theta=\sqrt{-\varphi} \rho \cos \theta-\frac{\varphi^{\prime}}{2 \sqrt{-\varphi}} \frac{\rho \sin \theta}{\sqrt{-\varphi}} \\
\rho^{\prime} \cos \theta-\rho \theta^{\prime} \sin \theta=\varphi \frac{\rho \sin \theta}{\sqrt{-\varphi}} .
\end{array}\right.
$$

From the above equation system (4), we have

$$
\begin{aligned}
\rho \theta^{\prime} & =\sqrt{-\varphi} \rho \cos ^{2} \theta-\frac{\varphi^{\prime}}{2 \sqrt{-\varphi}} \frac{\rho \sin \theta \cos \theta}{\sqrt{-\varphi}}-\varphi \frac{\rho \sin ^{2} \theta}{\sqrt{-\varphi}} \\
& =\rho \sqrt{-\varphi} \cos ^{2} \theta+\rho \sqrt{-\varphi} \sin ^{2} \theta+\frac{\varphi^{\prime}}{2 \varphi} \rho \sin \theta \cos \theta,
\end{aligned}
$$

therefore $\theta^{\prime}=\sqrt{-\varphi}+\frac{\varphi^{\prime}}{2 \varphi} \sin \theta \cos \theta$. From Equation (3), we get

$$
f(t)=c \exp \left(\int \sqrt{-\varphi} \frac{\cos \theta}{\sin \theta} d t\right), \quad(c \in \mathbb{R}) .
$$

At last, the solution of the Equation (1) can be expressed by

$$
F(t)=c \exp \left(\int\left[\sqrt{-\varphi} \frac{\cos \theta}{\sin \theta}+\frac{1}{2} b(t)\right] d t\right) \quad(c \in \mathbb{R}),
$$

where $\theta$ satisfies

$$
\theta^{\prime}=\sqrt{-\varphi}+\frac{\varphi^{\prime}}{2 \varphi} \sin \theta \cos \theta
$$

\section{Main Results}

Let $x(u, v): \mathbb{M} \rightarrow \mathbb{Q}^{3} \subset \mathbb{E}_{1}^{4}$ be an oriented and connected 2-dimensional differential manifold with isothermal parameters and induced metric as follows

$$
\langle d x, d x\rangle=2 e^{w}\left(d u^{2}+d v^{2}\right)=e^{w}(d z \otimes d \bar{z}+d \bar{z} \otimes d z)
$$

where $z=u+i v$. 
Denoting $x_{z}=\partial_{z} x=\partial x / \partial z, x_{\bar{z}}=\partial_{\bar{z}} x=\partial x / \partial \bar{z}$ and using the Cauchy-Riemann operators

$$
\partial_{z}=\frac{1}{2}\left(\frac{\partial}{\partial u}-i \frac{\partial}{\partial v}\right), \quad \partial_{\bar{z}}=\frac{1}{2}\left(\frac{\partial}{\partial u}+i \frac{\partial}{\partial v}\right),
$$

we have

$$
\langle x, x\rangle=\left\langle x, x_{z}\right\rangle=\left\langle x, x_{\bar{z}}\right\rangle=\left\langle x_{z}, x_{z}\right\rangle=\left\langle x_{\bar{z}}, x_{\bar{z}}\right\rangle=0, \quad\left\langle x_{z}, x_{\bar{z}}\right\rangle=e^{w} .
$$

After direct calculations by (6), we have easily

$$
\left\{\begin{array}{l}
\left\langle x_{z}, x_{z z}\right\rangle=\left\langle x_{z}, x_{z \bar{z}}\right\rangle=\left\langle x_{\bar{z}}, x_{\overline{z z}}\right\rangle=\left\langle x_{\bar{z}}, x_{z \bar{z}}\right\rangle=\left\langle x, x_{z z}\right\rangle=\left\langle x, x_{\overline{z z}}\right\rangle=0, \\
\left\langle x_{\bar{z}}, x_{z z}\right\rangle=e^{w_{1}} w_{z}, \quad\left\langle x_{z}, x_{\overline{z z}}\right\rangle=e^{w_{w}} w_{\bar{z}}, \quad\left\langle x, x_{z \bar{z}}\right\rangle=-e^{w} .
\end{array}\right.
$$

The Laplace operator $\Delta$ and the Gaussian curvature $K$ can be given by respectively

$$
\Delta=2 e^{-w} \partial_{z} \partial_{\bar{z}}, \quad K=-e^{-w} w_{z \bar{z}}
$$

Defining a new surface by the Laplacian $\Delta x$ and $x(u, v)$ as follows

$$
y=y(u, v)=-\frac{1}{2} \Delta x-\frac{1}{8}\langle\Delta x, \Delta x\rangle x
$$

which is said to be the associate surface of $x(u, v)$ in [3].

Obviously, we have

$$
\langle x, y\rangle=1, \quad\langle y, y\rangle=0, \quad\left\langle y, x_{z}\right\rangle=\left\langle y, x_{\bar{z}}\right\rangle=0
$$

In $\mathbb{Q}^{3}$, the vector fields $\left\{x, y,\left(2 e^{w}\right)^{-1 / 2} x_{u},\left(2 e^{w}\right)^{-1 / 2} x_{v}\right\}$ form an asymptotic orthogonal frame of $x(u, v)$. Based on above information, the structure equations and the integrable conditions of $x(u, v)$ are obtained as

$$
\left\{\begin{array}{l}
x_{z z}=w_{z} x_{z}+\varphi x \\
x_{z \bar{z}}=\lambda x-e^{w} y \\
y_{z}=-\lambda e^{-w} x_{z}-\varphi e^{-w} x_{\bar{z}}
\end{array}\right.
$$

and

$$
\left\{\begin{aligned}
\lambda & =-\frac{1}{2} e^{w} K \\
\varphi_{\bar{z}} & =-\frac{1}{2} e^{w} K_{z}
\end{aligned}\right.
$$

By (10) and (11), we obtain

$$
\left\{\begin{aligned}
H & =\lambda e^{-w}=-\frac{1}{2} K \\
\Delta x & =2 H-2 y
\end{aligned}\right.
$$

where $H=\frac{1}{2}\langle\Delta x, y\rangle$ is the mean curvature of $x(u, v)$.

Putting $\xi=\xi(u, v)=\xi(z, \bar{z})=2 x_{z}=\frac{\partial x}{\partial u}-i \frac{\partial x}{\partial v}$, according to (5), we have

$$
\xi^{2}=x_{u}^{2}-x_{v}^{2}-2 i x_{u} \cdot x_{v}=0
$$

and

$$
|\xi|^{2}=\xi \bar{\xi}=\left|\frac{\partial x}{\partial u}\right|^{2}+\left|\frac{\partial x}{\partial v}\right|^{2}=4 e^{w}
$$


Making use of the frame $\{\xi, \bar{\xi}, x, y\}$, then the structure equations (10) of $x(u, v)$ are rewritten by

$$
\left\{\begin{array}{l}
\frac{\partial \xi}{\partial z}=\xi_{z}=w_{z} \xi+2 \varphi x \\
\frac{\partial \xi}{\partial \bar{z}}=\xi \bar{z}=2 H e^{w} x-2 e^{w} y \\
2 \frac{\partial y}{\partial z}=2 y_{z}=-H \xi-\varphi e^{-w} \bar{\xi}
\end{array}\right.
$$

The integrable conditions (11) can be rewritten by

$$
\left\{\begin{aligned}
w_{z \bar{z}} & =2 H e^{w}, \\
\varphi_{\bar{z}} & =H_{z} e^{w}
\end{aligned}\right.
$$

or

$$
\left\{\begin{array}{c}
2 H+K=0, \\
\varphi_{\bar{z}}=H_{z} e^{w} .
\end{array}\right.
$$

It is not difficult to find the following conclusion by (17).

Proposition 1. Let $x(u, v): \mathbb{M} \rightarrow \mathbb{Q}^{3} \subset \mathbb{E}_{1}^{4}$ be a surface. Then it is maximal if and only if it is flat.

Assuming that $\xi=\left(\xi_{1}, \xi_{2}, \xi_{3}, \xi_{4}\right)$, then $\xi_{1}^{2}+\xi_{2}^{2}+\xi_{3}^{2}-\xi_{4}^{2}=0$. Choosing complex functions $f$, $g$ and $\rho$ such that

$$
\left\{\begin{array}{l}
\xi_{1}=\rho(f+g), \\
\xi_{2}=-i \rho(f-g), \\
\xi_{3}=\rho(1-f g), \\
\xi_{4}=\rho(1+f g),
\end{array}\right.
$$

thus, we have

$$
\xi=\xi(z, \bar{z})=(\rho(f+g),-i \rho(f-g), \rho(1-f g), \rho(1+f g)) .
$$

At the same time, we have

$$
\left\{\begin{array}{l}
\rho=\frac{1}{2 f g}\left(-\xi_{3}+\xi_{4}\right), \\
f=\frac{1}{2 \rho}\left(\xi_{1}+i \xi_{2}\right), \\
g=\frac{1}{2 \rho}\left(\xi_{1}-i \xi_{2}\right) .
\end{array}\right.
$$

Furthermore, by a direct calculation from (15), we have

$$
\xi_{z z}=\left(w_{z z}+\varphi-\varphi_{z} \varphi^{-1} w_{z}\right) \xi+\left(w_{z}+\varphi_{z} \varphi^{-1}\right) \xi_{z} .
$$

Together with the Equations (19) and (21), we have

$$
\left\{\begin{array}{l}
\frac{\partial^{2}[\rho(f+g)]}{\partial z^{2}}=\left(w_{z z}+\varphi-\varphi_{z} \varphi^{-1} w_{z}\right) \rho(f+g)+\left(w_{z}+\varphi_{z} \varphi^{-1}\right)[\rho(f+g)]_{z} \\
\frac{\partial^{2}[\rho(f-g)]}{\partial z^{2}}=\left(w_{z z}+\varphi-\varphi_{z} \varphi^{-1} w_{z}\right) \rho(f-g)+\left(w_{z}+\varphi_{z} \varphi^{-1}\right)[\rho(f-g)]_{z} \\
\frac{\partial^{2}[\rho(1-f g)]}{\partial z^{2}}=\left(w_{z z}+\varphi-\varphi_{z} \varphi^{-1} w_{z}\right) \rho(1-f g)+\left(w_{z}+\varphi_{z} \varphi^{-1}\right)[\rho(1-f g)]_{z} \\
\frac{\partial^{2}[\rho(1+f g)]}{\partial z^{2}}=\left(w_{z z}+\varphi-\varphi_{z} \varphi^{-1} w_{z}\right) \rho(1+f g)+\left(w_{z}+\varphi_{z} \varphi^{-1}\right)[\rho(1+f g)]_{z}
\end{array}\right.
$$


through simplification, we know $\rho, f$ and $g$ satisfy the following equation system

$$
\left\{\begin{aligned}
\frac{\partial^{2} \rho}{\partial z^{2}} & =\left(w_{z z}+\varphi-\varphi_{z} \varphi^{-1} w_{z}\right) \rho+\left(w_{z}+\varphi_{z} \varphi^{-1}\right) \rho_{z} \\
\frac{\partial^{2}(\rho f)}{\partial z^{2}} & =\left(w_{z z}+\varphi-\varphi_{z} \varphi^{-1} w_{z}\right)(\rho f)+\left(w_{z}+\varphi_{z} \varphi^{-1}\right)(\rho f)_{z} \\
\frac{\partial^{2}(\rho g)}{\partial z^{2}} & =\left(w_{z z}+\varphi-\varphi_{z} \varphi^{-1} w_{z}\right)(\rho g)+\left(w_{z}+\varphi_{z} \varphi^{-1}\right)(\rho g)_{z} \\
\frac{\partial^{2}(\rho f g)}{\partial z^{2}} & =\left(w_{z z}+\varphi-\varphi_{z} \varphi^{-1} w_{z}\right)(\rho f g)+\left(w_{z}+\varphi_{z} \varphi^{-1}\right)(\rho f g)_{z}
\end{aligned}\right.
$$

indeed, $\rho, \rho f, \rho g$ and $\rho f g$ are the solutions of Equation (21).

Conversely, if we choose the complex function $\rho, f$ and $g$ which satisfy Equation (21), then by letting

$$
\xi=\xi(z, \bar{z})=(\rho(f+g),-i \rho(f-g), \rho(1-f g), \rho(1+f g))
$$

we have $\xi_{\bar{z}}=\bar{\xi}_{z}$. From Lemma 1 , we get $2 x_{z}=\xi, \xi^{2}=0$, thus Equation (21) takes a real surface $x(u, v)$ with isothermal parameter.

Considering the non-degenerate condition of the surface $x(u, v)$, we know $|\rho||f-\bar{g}| \neq 0$, then the representation of $x(u, v)$ can be achieved by Lemma 1, easily.

Theorem 1. Let $x(u, v): \mathbb{M} \rightarrow \mathbb{Q}^{3} \subset \mathbb{E}_{1}^{4}$ be a surface with isothermal parameters. Then $x(u, v)$ can be expressed locally as

$$
x(u, v)=\left(\int \operatorname{Re}[\rho(f+g) d z], \int \operatorname{Re}[-i \rho(f-g) d z], \int \operatorname{Re}[\rho(1-f g) d z], \int \operatorname{Re}[\rho(1+f g) d z]\right),
$$

where $|\rho||f-\bar{g}| \neq 0$ and $\rho, \rho f, \rho g$ and $\rho$ fg are the solutions of (23) for some holomorphic function $\varphi$.

For the maximal surface with isothermal parameters $(u, v)$ in $\mathbb{Q}^{3}$, from Proposition 1 , we know that $x(u, v)$ is flat and $\varphi_{\bar{z}} \equiv 0$ by (17). Without loss of generality, we can choose $(u, v)$ such that $e^{w(u, v)}=1$. Using Theorem 1 and (23), we have

$$
\left\{\begin{aligned}
\frac{\partial^{2} \rho}{\partial z^{2}} & =\varphi \rho+\varphi_{z} \varphi^{-1} \rho_{z} \\
\frac{\partial^{2}(\rho f)}{\partial z^{2}} & =\varphi(\rho f)+\varphi_{z} \varphi^{-1}(\rho f)_{z} \\
\frac{\partial^{2}(\rho g)}{\partial z^{2}} & =\varphi(\rho g)+\varphi_{z} \varphi^{-1}(\rho g)_{z} \\
\frac{\partial^{2}(\rho f g)}{\partial z^{2}} & =\varphi(\rho f g)+\varphi_{z} \varphi^{-1}(\rho f g)_{z}
\end{aligned}\right.
$$

Therefore, we can obtain the following result for maximal surfaces in the lightlike cone.

Theorem 2. Let $x(u, v): \mathbb{M} \rightarrow \mathbb{Q}^{3} \subset \mathbb{E}_{1}^{4}$ be a maximal surface with isothermal parameters. Then $x(u, v)$ can be represented locally as

$$
x(u, v)=\left(\int \operatorname{Re}[\rho(f+g) d z], \int \operatorname{Re}[-i \rho(f-g) d z], \int \operatorname{Re}[\rho(1-f g) d z], \int \operatorname{Re}[\rho(1+f g) d z]\right),
$$

where $|\rho||f-\bar{g}| \neq 0$ and $\rho, \rho f, \rho g$ and $\rho$ fg are the solutions of (24) for some holomorphic function $\varphi$.

Remark 1. Denoting $h_{i j}$ the quantities of the second fundamental form of $x(u, v)$ defined by

$$
h_{i j}=\left(2 e^{w}\right)^{-1}\left\langle x_{u^{i} u^{j}}, y\right\rangle,
$$


where $u^{1}=u, u^{2}=v$.

From [3], we know that

$$
\varphi=\frac{1}{2} e^{w}\left(h_{11}-h_{22}-2 i h_{12}\right)
$$

Obviously, for prescribed holomorphic function $\varphi$, it is not difficult to get the solutions of Equation (23) by Lemma 2.

Corollary 1. Let $x(u, v): \mathbb{M} \rightarrow \mathbb{Q}^{3} \subset \mathbb{E}_{1}^{4}$ be a surface with induced metric

$$
d s^{2}=d u^{2}+d v^{2}
$$

- When

$$
\left\{\begin{array}{l}
\rho=\frac{-i}{2} e^{-\frac{i}{2}(z-\bar{z})}=\frac{-i}{2} e^{v} \\
f=i e^{-i \bar{z}}=i e^{-v} e^{-i u} \\
g=i e^{i z}=i e^{-v} e^{i u}
\end{array}\right.
$$

the surface $x(u, v)$ can be written as

$$
x(u, v)=(\sin u, \cos u, \sinh v, \cosh v),
$$

which is a maximal surface with isothermal parameters;

- When

$$
\left\{\begin{array}{l}
\rho=\frac{-i}{2} e^{-\frac{i}{2}(z-\bar{z})}=\frac{-i}{2} e^{v} \\
f=-i e^{-i \bar{z}}=-i e^{-v} e^{-i u} \\
g=-i e^{i z}=-i e^{-v} e^{i u}
\end{array}\right.
$$

the surface $x(u, v)$ can be written as

$$
x(u, v)=(-\sin u,-\cos u, \sinh v, \cosh v),
$$

which is a maximal surface with isothermal parameters.

Proof. Firstly, from (18) and (25), we know

$$
\left\{\begin{array}{l}
\xi_{1}=\rho(f+g)=\cos u \\
\xi_{2}=-i \rho(f-g)=-\sin u \\
\xi_{3}=\rho(1-f g)=-i \cosh v \\
\xi_{4}=\rho(1+f g)=-i \sinh v
\end{array}\right.
$$

Similarly, by (18) and (26), we have

$$
\left\{\begin{array}{l}
\xi_{1}=\rho(f+g)=-\cos u \\
\xi_{2}=-i \rho(f-g)=\sin u \\
\xi_{3}=\rho(1-f g)=-i \cosh v \\
\xi_{4}=\rho(1+f g)=-i \sinh v
\end{array}\right.
$$

According to (27), (28) and Theorem 1, the surface $x(u, v)=\int \operatorname{Re} \xi d z$ can be expressed. Meanwhile, they are all maximal surfaces in $\mathbb{Q}^{3}$ with isothermal parameters $(u, v)$. 
Corollary 2. Let $x(u, v): \mathbb{M} \rightarrow \mathbb{Q}^{3} \subset \mathbb{E}_{1}^{4}$ be a surface with induced metric

$$
d s^{2}=e^{2 \lambda u+2 \mu v}\left(d u^{2}+d v^{2}\right), \quad(\lambda, \mu \in \mathbb{R}) .
$$

- When

$$
\left\{\begin{array}{l}
\rho=\frac{1}{2}(\lambda-i \mu-i) e^{\frac{\lambda}{2}(z+\bar{z})+\frac{-i \mu}{2}(z-\bar{z})} e^{-\frac{i}{2}(z-\bar{z})}=\frac{1}{2}(\lambda-i \mu-i) e^{\lambda u+\mu v} e^{v}, \\
f=i e^{-i \bar{z}}=i e^{-v} e^{-i u}, \\
g=\frac{-i \lambda-\mu+1}{\lambda-i \mu-i} e^{i z}=\frac{-i \lambda-\mu+1}{\lambda-i \mu-i} e^{-v} e^{i u},
\end{array}\right.
$$

the surface $x(u, v)$ can be expressed as

$$
x(u, v)=e^{\lambda u+\mu v}(\sin u, \cos u, \sinh v, \cosh v),
$$

which is a maximal surface with isothermal parameters;

- When

$$
\left\{\begin{array}{l}
\rho=(\lambda-i \mu) e^{\frac{\lambda}{2}(z+\bar{z})+\frac{-i \mu}{2}(z-\bar{z})}=(\lambda-i \mu) e^{\lambda u+\mu v} \\
f=\frac{2+(\lambda-i \mu) z}{2(\lambda-i \mu)}=\frac{2+(\lambda-i \mu)(u+i v)}{2(\lambda-i \mu)} \\
g=\frac{\bar{z}}{2}=\frac{u-i v}{2}
\end{array}\right.
$$

the surface $x(u, v)$ can be expressed as

$$
x(u, v)=e^{\lambda u+\mu v}\left(u, v, 1-\frac{1}{4}\left(u^{2}+v^{2}\right), 1+\frac{1}{4}\left(u^{2}+v^{2}\right)\right),
$$

which is a maximal surface with isothermal parameters.

Proof. By using (18) and (29), we obtain

$$
\left\{\begin{array}{l}
\xi_{1}=\rho(f+g)=e^{\lambda u+\mu v}[(\lambda \sin u+\cos u)-i \mu \sin u], \\
\xi_{2}=-i \rho(f-g)=e^{\lambda u+\mu v}[(\lambda \cos u-\sin u)-i \mu \cos u], \\
\xi_{3}=\rho(1-f g)=e^{\lambda u+\mu v}[\lambda \sinh v-i(\mu \sinh v+\cosh v)], \\
\xi_{4}=\rho(1+f g)=e^{\lambda u+\mu v}[\lambda \cosh v-i(\mu \cosh v+\sinh v)] .
\end{array}\right.
$$

And from (18) and (30), we obtain

$$
\left\{\begin{array}{l}
\xi_{1}=\rho(f+g)=e^{\lambda u+\mu v}(1+(\lambda-i \mu) u) \\
\xi_{2}=-i \rho(f-g)=e^{\lambda u+\mu v}(-i+(\lambda-i \mu) v) \\
\xi_{3}=\rho(1-f g)=e^{\lambda u+\mu v}\left(\lambda-i \mu-\frac{1}{4}(\lambda-i \mu)\left(u^{2}+v^{2}\right)-\frac{1}{2}(u-i v)\right) \\
\xi_{4}=\rho(1+f g)=e^{\lambda u+\mu v}\left(\lambda-i \mu+\frac{1}{4}(\lambda-i \mu)\left(u^{2}+v^{2}\right)+\frac{1}{2}(u-i v)\right) .
\end{array}\right.
$$

According to (31), (32) and Theorem 1, the surface $x(u, v)=\int \operatorname{Re} \xi d z$ can be represented. Meanwhile, they are all maximal surfaces in $\mathbb{Q}^{3}$ with isothermal parameters $(u, v)$ by considering the integrable condition and the Cauchy-Riemann operator for $\omega=\lambda u+\mu v$. 
Corollary 3. Let $x(u, v): \mathbb{M} \rightarrow \mathbb{Q}^{3} \subset \mathbb{E}_{1}^{4}$ be a surface with induced metric

$$
d s^{2}=e^{2 \lambda u v}\left(d u^{2}+d v^{2}\right), \quad(\lambda \in \mathbb{R}) .
$$

- When

$$
\left\{\begin{array}{l}
\rho=\frac{1}{2}(-i \lambda z-i) e^{\frac{-i \lambda}{4}\left(z^{2}-\bar{z}^{2}\right)} e^{-\frac{i}{2}(z-\bar{z})}=\frac{1}{2}(\lambda v-i \lambda u-i) e^{\lambda u v} e^{v}, \\
f=i e^{-i \bar{z}}=i e^{-v} e^{-i u} \\
g=\frac{-\lambda z+1}{-i \lambda z-i} e^{i z}=\frac{-i \lambda v-\lambda u+1}{\lambda v-i \lambda u-i} e^{-v} e^{i u} .
\end{array}\right.
$$

the surface $x(u, v)$ can be represented as

$$
x(u, v)=e^{\lambda u v}(\sin u, \cos u, \sinh v, \cosh v),
$$

which is a maximal surface with isothermal parameters;

- When

$$
\left\{\begin{array}{l}
\rho=-i \lambda z e^{\frac{-i \lambda}{4}\left(z^{2}-\bar{z}^{2}\right)}=\lambda(v-i u) e^{\lambda u v} \\
f=\frac{2-i \lambda z^{2}}{-2 i \lambda z}=\frac{2+\lambda(v-i u)(u+i v)}{2 \lambda(v-i u)} \\
g=\frac{\bar{z}}{2}=\frac{u-i v}{2}
\end{array}\right.
$$

the surface $x(u, v)$ can be represented as

$$
x(u, v)=e^{\lambda u v}\left(u, v, 1-\frac{1}{4}\left(u^{2}+v^{2}\right), 1+\frac{1}{4}\left(u^{2}+v^{2}\right)\right),
$$

which is a maximal surface with isothermal parameters.

Proof. By (18) and (33), we get

$$
\left\{\begin{array}{l}
\xi_{1}=\rho(f+g)=e^{\lambda u v}[(\lambda v \sin u+\cos u)-i \lambda u \sin u], \\
\xi_{2}=-i \rho(f-g)=e^{\lambda u v}[(\lambda v \cos u-\sin u)-i \lambda u \cos u], \\
\xi_{3}=\rho(1-f g)=e^{\lambda u v}[\lambda v \sinh v-i(\lambda u \sinh v+\cosh v)] \\
\xi_{4}=\rho(1+f g)=e^{\lambda u v}[\lambda v \cosh v-i(\lambda u \cosh v+\sinh v)] .
\end{array}\right.
$$

And from (18) and (34), we have

$$
\left\{\begin{array}{l}
\xi_{1}=\rho(f+g)=e^{\lambda u v}(1+\lambda u(v-i u)) \\
\xi_{2}=-i \rho(f-g)=e^{\lambda u v}(-i+\lambda v(v-i u)), \\
\xi_{3}=\rho(1-f g)=e^{\lambda u v}\left(\lambda(v-i u)-\frac{\lambda}{4}\left(u^{2}+v^{2}\right)(v-i u)-\frac{1}{2}(u-i v)\right), \\
\xi_{4}=\rho(1+f g)=e^{\lambda u v}\left(\lambda(v-i u)+\frac{\lambda}{4}\left(u^{2}+v^{2}\right)(v-i u)+\frac{1}{2}(u-i v)\right) .
\end{array}\right.
$$

According to (35), (36) and Theorem 1, the surface $x(u, v)=\int \operatorname{Re} \xi d z$ can be obtained, and they are all maximal surfaces in $\mathbb{Q}^{3}$ with isothermal parameters $(u, v)$ by using the Cauchy-Riemann operator and the integrable condition for $\omega=\lambda u v$. 
Remark 2. According to (9), the associated surface y of maximal surface $x$ can be achieved. From Theorem 2.2 of [3], we know that $y(u, v)$ is also maximal in $\mathbb{Q}^{3}$. In fact, the surfaces in Corollary 1 are associated maximal surfaces. In a similar way, we can get more examples for maximal surfaces in $\mathbb{Q}^{3}$.

\section{Example}

In this section, we present some maximal surfaces by the conclusion in Proposition 1.

Example 1. For any real number $\lambda, \mu \in \mathbb{R}$, the surface

$$
x(u, v)=\left(\tan \frac{u}{2}\right)^{\lambda} \frac{e^{\mu v}}{\sin u}(\sin u \sin v, \sin u \cos v, \cos u, 1)
$$

is flat and maximal in $\mathbb{Q}^{3}$, whose metric is

$$
d s^{2}=f^{2} e^{2 \mu v}\left(d u^{2}+\sin ^{2} u d v^{2}\right),
$$

here $f=f(u)=\left(\tan \frac{u}{2}\right)^{\lambda} \frac{1}{\sin u}$.

Example 2. For any real number $\lambda, \mu \in \mathbb{R}$, the surface

$$
x(u, v)=\left(\tanh \frac{u}{2}\right)^{\lambda} \frac{e^{\mu v}}{\sinh u}(1, \sinh u \sin v, \sinh u \cos v, \cosh u)
$$

is flat and maximal in $\mathbb{Q}^{3}$, whose metric is

$$
d s^{2}=f^{2} e^{2 \mu v}\left(d u^{2}+\sinh ^{2} u d v^{2}\right)
$$

here $f=f(u)=\left(\tanh \frac{u}{2}\right)^{\lambda} \frac{1}{\sinh u}$.

Example 3. For any real number $\lambda, \mu \in \mathbb{R}$, the surface

$$
x(u, v)=\frac{e^{\lambda \arcsin (\tanh u)} e^{\mu v}}{\cosh u}(1, \sinh u, \cosh u \sinh v, \cosh u \cosh v)
$$

is flat and maximal in $\mathbb{Q}^{3}$, whose metric is

$$
d s^{2}=f^{2} e^{2 \mu v}\left(d u^{2}+\cosh ^{2} u d v^{2}\right),
$$

here $f=f(u)=\frac{e^{\lambda \arcsin (\tanh u)}}{\cosh u}$.

Remark 3. The parameters in the above examples are not isothermal parameters.

Author Contributions: H.L. set up the problem, J.Q. and X.F. computed the details. All authors have read and agree to the published version of the manuscript.

Funding: The authors were supported by NSFC (No.11801065,11371080) and the Fundamental Research Funds for the Central Universities (N2005012).

Acknowledgments: We thank the referee for the careful review and the valuable comments to improve the paper.

Conflicts of Interest: The authors declare no conflict of interest. 


\section{References}

1. Chen, W.H. General Weierstrass formula of surfaces in Euclidean 3-space $\mathbb{R}^{3}$. Acta Math. Sin. 1987, 30, 361-367.

2. Wang, C.P. Weierstrass representations of Laguerre minimal surfaces in $\mathbb{R}^{3}$. Results Math. 2008, 52, 399-408. [CrossRef]

3. Liu, H.L. Surfaces in the lightlike cone. J. Math. Anal. Appl. 2007, 325, 1171-1181. [CrossRef]

4. Liu, H.L. Representation of surfaces in 3-dimensional lightlike cone. Bull. Belg. Math. Soc. Simon Stevin. 2011, 18, 737-748. [CrossRef]

5. Liu, H.L. Weierstrass type representation for marginally trapped surfaces in Minkowski 4-space. Math. Phys. Anal. Geom. 2013, 16, 171-178. [CrossRef]

6. Liu, H.L.; Jung, S.D. Hypersurfaces in lightlike cone. J. Geom. Phys. 2008, 58, 913-922. [CrossRef]

7. Chen, B.Y. Black holes, marginally trapped surfaces and quasi-minimal surfaces. Tamkang J. Math. 2009, 40, 1-26. [CrossRef]

8. Chen, B.Y. Marginally trapped surfaces and Kaluza-Klein theory. Int. Electron. J. Geom. 2009, 2, 1-16.

Publisher's Note: MDPI stays neutral with regard to jurisdictional claims in published maps and institutional affiliations.

(C) 2020 by the authors. Licensee MDPI, Basel, Switzerland. This article is an open access article distributed under the terms and conditions of the Creative Commons Attribution (CC BY) license (http:/ / creativecommons.org/licenses/by/4.0/). 